\title{
Bioinformatics analysis and biological experiments support an emerging role of ELK3 in glioma
}

\author{
Jiang-Tao Dong, Hui Xv, Fu-Lei Chen, Shi-Long Wang, Yang Wang, Li-Cang Zhu
}

Department of Neurosurgery, First Affiliated Hospital, School of Medicine, Shihezi University, Shihezi, China

Submitted: 29 December 2020; Accepted: 21 July 2021

Online publication: 7 August 2021

Arch Med Sci

DOI: https://doi.org/ 10.5114/aoms/140398

Copyright $\odot 2022$ Termedia \& Banach

\begin{abstract}
Introduction: Glioma is a malignant tumor that recurs easily. This study aimed to investigate the biological effect of ELK3 in epithelial-mesenchymal transition (EMT) and its prognostic value.

Material and methods: Bioinformatics analyses were performed to determine the expression and prognostic value of ELK3. The biological properties of glioma cells (A172 and LN229) were evaluated by Cell Counting Kit-8, plate cloning and transwell assays. The Gene Expression Profiling Interactive Analysis (GEPIA) website was used to analyze the relationship between ELK3 expression and EMT-related markers. Western blotting was applied to detect the protein levels.

Results: ELK3 is highly expressed in glioma and significantly associated with poor prognosis $(p<0.05)$, presenting promising value in the diagnosis and prognostic prediction of glioma. Further biological assays revealed that cell viability was notably reduced after down-regulating ELK3 $(p<0.01)$. The numbers of colonies formed by A172 (145.30 \pm 12.86 vs. $300.30 \pm 11.68)$ and LN229 (125.70 \pm 4.04 vs. $256.00 \pm 20.07)$ cells were decreased after transfection of si-ELK3 $(p<0.01)$. The numbers of invaded and migrated glioma cells in the si-ELK3 group were decreased by about half $(p<0.01)$. Moreover, ELK3 was positively correlated with $\mathrm{N}$-cadherin $(R=0.21)$, Snail1 $(R=0.43)$, Slug $(R=0.6)$ and vimentin $(R=0.35)$, and depletion of ELK3 reduced the levels of these EMT-related markers more than a half $(p<0.01)$.

Conclusions: Our observations illustrated that ELK3 was highly expressed in glioma and played a promoting role in the growth and mobility of glioma cells partly by modulating EMT progression.
\end{abstract}

Key words: ELK3, epithelial-mesenchymal transition, glioma, migration, prognosis.

\section{Introduction}

Gliomas are common, highly malignant primary tumors of the brain and spinal cord, with a median survival time of less than 16 months after diagnosis [1]. Histologically, they have the properties of normal glial cells and are usually named based on these similarities [2]. Despite the continuous improvement of surgical resection, radiotherapy, chemotherapy and other treatment methods, the survival rate of glioma patients is still low $[3,4]$. This is mainly due to its highly aggressive growth pattern, prominent tumor angiogenesis, and high resistance to treatment [5]. As the prognosis of glioma patients is dismal and the therapeutic effect is poor, it is particularly

\author{
Corresponding author: \\ Li-Cang Zhu \\ Department of Neurosurgery \\ First Affiliated Hospital \\ School of Medicine \\ Shihezi University \\ Shihezi, 832000, China \\ E-mail: \\ zhulicang_2002@163.com
}


urgent to explore new markers for early diagnosis and treatment of glioma. At the same time, elucidating the molecular mechanism of glioma may also provide a new direction for the treatment of glioma.

The biological functions of E-twenty-six (ETS) transcription factor ELK3 (ELK3) in tumors have been preliminarily validated by several researchers. For example, ELK3 has been found to be expressed in lymphatic endothelial cells and promote the development and metastasis of breast cancer [6]. Furthermore, ELK3 can contribute to the invasion and migration of liver cancer stem cells by regulating HIF-1 $\alpha$ [7]. Furthermore, it has been reported that mice lacking ELK3 protein developed smaller tumors, which were not able to become vascularized and oxygenated, suggesting that ELK3 holds a primary role in tumorigenicity [8]. Hence, the existing literature reveals that ELK3 plays an important role in the growth and mobility of tumor cells. However, reports on the function and mechanism of ELK3 in glioma are still rare.

Epithelial-mesenchymal transition (EMT) is a multistep and dynamic process during which epithelial cells' phenotypic features are lost [9]. It plays an important role in generating and maintaining the malignant characteristics of primary glioma as well as grade II-III glioma [10]. Certain transcription factors such as Slug and Snail have been reported to be involved in EMT, and promote the migration and invasion of glioma cells [11]. Vimentin and $\mathrm{N}$-cadherin are critical mesenchymal biomarkers of the EMT process [12]. It has been reported that ELK3 contributes to the process of liver fibrosis via modulating EMT [9]. In addition, ELK3 is identified as a partner of ZEB1 to suppress the expression of E-cadherin in triple-negative breast cancer cells [13]. Whether ELK3 involved in EMT process in glioma remains unknown.

In this study, the intention was to investigate the expression and the prognostic value of ELK3 using data on glioma from publicly available databases. Moreover, the malignant behaviors of glioma cells and the expression of EMT-related proteins were examined after depletion of ELK3 to reveal the biological functions of ELK3 in glioma.

\section{Material and methods}

\section{Bioinformatics analyses}

Data of 168 tumor samples and 110 normal samples downloaded from The Cancer Genome Atlas (TCGA) database and Genotype-Tissue Expression (GTEx) database were used to analyze the differential expression of ELK3. Receiver operating characteristic (ROC) curve analysis was performed to assess whether ELK3 was capable of discriminating glioma and normal samples. mRNAseq_325, mRNAseq_693 and mRNA_array_301 datasets were downloaded from Chinese Glioma Genome Atlas (CGGA) database and applied to detect the expression of ELK3 and its prognostic value in patients with different World Health Organization (WHO) tumor stages, patients' ages and recurrence status. The Gene Expression Profiling Interactive Analysis (GEPIA) website was applied to analyze the association between ELK3 expression and N-cadherin, Snail1, Slug or vimentin.

\section{Cell culture and transfection}

Shanghai Cell Bank of the Chinese Academy of Sciences (Shanghai, China) provided the glioma cell lines A172 and LN229. The A172 cell line was originally from the brain of a 53-year-old man with glioblastoma. It was not tumorigenic in immunosuppressed mice. The LN229 cell line was originally from a patient with right frontal parieto-occipital glioblastoma. This cell line displayed mutated p53 and possible homozygous deletions in the p16 and p14ARF tumor suppressor genes, and possessed a wild-type PTEN gene. These two cell lines are commonly used strains for studying glioma in vitro. The normal human astrocyte (NHA) cell line was obtained from ScienCell Research Laboratories and used as a control. All cell lines were cultured in Dulbecco Modified Eagle medium (DMEM) with antibiotics at $37^{\circ} \mathrm{C}$ with $5 \% \mathrm{CO}_{2}$. Si-ELK3\#1 and si-ELK3\#2 are siRNAs that were applied to down-regulate ELK3 expression, and si-con served as a corresponding control. All these oligonucleotides were synthesized by Sangon (Shanghai, China) and transfected into A172 and LN229 cells by Lipofectamine 2000 (Invitrogen, USA). The sequences of these siRNAs are listed below:

si-ELK3\#1: 5'-GCCACAATTAAGGACTCATTA-3'; si-ELK3\#2: 5'-TGGATCAGAAACATGAGCATT-3'; si-con: 5'-AATTCTCCGAACGTGTCACGT-3'.

\section{Quantitative real-time polymerase chain reaction (qRT-PCR)}

Total RNA from A172 and LN229 cells was extracted using TRIzol reagent (TaKaRa, China) based on the supplier's instructions. To quantify the expression of ELK3, we first synthesized cDNA from total RNA using the PrimeScript RT Reagent kit (TaKaRa, China). Then, qRT-PCR was performed utilizing SYBR Green Mix in a 7900 HT RT-PCR machine and GAPDH was used as an internal control for normalization. The relative expression of ELK3 was processed by the $2^{-\Delta \Delta c t}$ method. The sequences of the primers are listed below:

ELK3 forward: 5'-TTCTGACTCCGAGTCCACTGCT-3', reverse: 5'-AGCTCTGTCCAGACTGGGGATT-3'; GAPDH forward: 5'-GTCTCCTCTGACTTCAACAGCG-3', reverse: 5'-ACCACCCTGTTGCTGTAGCCAA-3'. 


\section{Western blotting}

A172 and LN229 cells were lysed, and the protein extracts were denatured. The proteins $(25 \mu \mathrm{g})$ were subjected to sodium dodecyl sulfate polyacrylamide gel electrophoresis, blotted onto polyvinylidene fluoride membranes and probed with specific primary antibodies against ELK3, N-cadherin, Snail, Slug, vimentin and GAPDH. Afterwards, the membranes were incubated with the secondary antibody and the signals of the objective proteins were visualized by enhanced chemiluminescence. Finally, the intensity of the bands was evaluated by Image J software.

\section{Cell Counting Kit-8 (CCK-8) assay}

For CCK-8 assay, the treated A172 and LN229 cells were implanted into a 96-well plate (1000 cells/well). The cells were routinely cultured in a carbon dioxide incubator at $37^{\circ} \mathrm{C}$, and the cell viability was monitored every $24 \mathrm{~h}$. Before each test, the CCK-8 solution $(10 \mu \mathrm{l})$ was loaded into each well based on the supplier's instruction. Then the plate was kept in the incubator at $37^{\circ} \mathrm{C}$ for $1.5 \mathrm{~h}$. After $1.5 \mathrm{~h}$ incubation, the optical density was examined at $450 \mathrm{~nm}$ wavelength.

\section{Plate colony formation assay}

To test the ability of the cells to form colonies, a total of 500 A172 and LN229 cells were seeded in a $60 \mathrm{~mm}$ petri dish and grew in the medium containing $10 \%$ fetal bovine serum (FBS). After 10-14 days of incubation, the emerging colonies were fixed, stained and manually counted. Each experiment was repeated at least three times.

\section{Cell invasion and migration assays}

For the cell invasion assay, a 24-well transwell chamber (Corning, New York, USA) coated with Matrigel (BD Bioscience, USA) was used. Briefly, $100 \mu \mathrm{L}$ of serum free medium was added to the upper chamber and incubated at $37^{\circ} \mathrm{C}$ for $1 \mathrm{~h}$. A172 and LN229 cells were implanted into the upper chamber at a concentration of $5 \times 10^{3}$ cells/ well, and $500 \mu \mathrm{L}$ of complete medium containing $10 \%$ FBS was put into the lower chamber. After cultivation for $24 \mathrm{~h}$ at $37^{\circ} \mathrm{C}$, the invaded cells were fixed and stained. Five fields were randomly selected to take photos. The steps of the migration assay were similar to the invasion assay except that the wells in the upper chamber did not require Matrigel.

\section{Statistical analyses}

SPSS22.0 and GraphPad Prism 8.0 software was used to process the experimental data, which were expressed as mean \pm standard devia- tion (SD). Student's t-test or analysis of variance (ANOVA) was applied to analyze the differences among groups. Kaplan-Meier analysis was applied to analyze the overall survival of glioma patients with different ELK3 expression and the log-rank test was used for comparison. The expression of ELK3 in patients with glioma was categorized as low expression or high expression according to the median value. A $p$-value less than 0.05 was considered to indicate a statistically significant difference.

\section{Results}

Expression, prognostic value and clinical significance of ELK3 in glioma

Expression data analyses of 168 tumor samples from TCGA and 110 normal samples from TCGA and GTEx illustrated that the expression of ELK3 was significantly higher in glioma samples than that in normal samples (Figure $1 \mathrm{~A}$ ). Moreover, the results from $\mathrm{ROC}$ analysis displayed that the area under the curve (AUC) was 0.9995 ( $p<0.001$ ), indicating that ELK3 expression was of great diagnostic value in distinguishing glioma from normal samples (Figure 1 B). Survival analysis showed that the overall survival of glioma patients in the high ELK3 expression group was shorter than that of the low ELK3 expression group (Figure 1 C).

Next, the expression of ELK3 in patients with different WHO tumor stages, ages, and recurrence status was analyzed based on the mRNAseq_325, mRNAseq_693 and mRNA_array_301 datasets from the CGGA database. mRNAseq_325 and mRNAseq_693 are RNA-seq datasets, while mRNA_array_301 is a microarray dataset. The sample numbers of these 3 datasets are presented in Table I. The results displayed that the expression of ELK3 in WHO tumor grade IV was higher than that in WHO tumor grades II and III and the difference was statistically significant (Figure $2 \mathrm{~A}-\mathrm{C}$, $p<0.001$ ). Additionally, the data from the mRNAseq_325 dataset showed that ELK3 expression was higher in the glioma patients over 42 years old (Figure 2 D, $p<0.001$ ). Similarly, the data from the mRNA_array_301 dataset displayed that ELK3 expression was higher in the glioma patients older than 43 years old compared with patients younger than 43 years (Figure $2 \mathrm{E}, p=0.029$ ), indicating that ELK3 tended to present a higher level in older people. Moreover, the data from the mRNAseq_693 dataset illustrated that ELK3 expression was higher in patients with recurrent glioma than that in patients with primary glioma (Figure $2 \mathrm{~F}$, $p=0.003)$. The prognostic analysis revealed that high expression of ELK3 led to a poor survival rate in the WHO II and III tumor grades (Figure $2 \mathrm{G}-\mathrm{I}$ ). In addition, high expression of ELK3 resulted in 
A

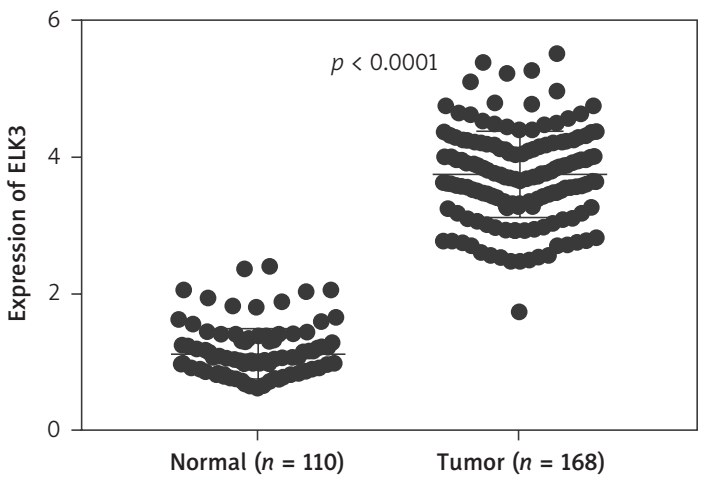

C

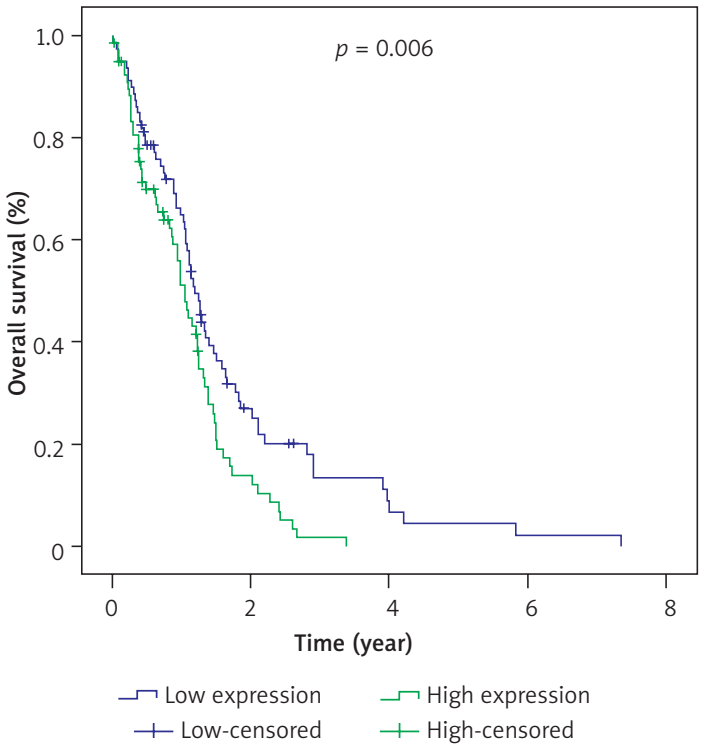

a poor prognosis in patients with primary or recurrent glioma, which was statistically significant (Figure $2 \mathrm{~J}-\mathrm{Q}, p<0.05$ ). All these results suggested that ELK3 is closely related to the progression of glioma and is a potential biomarker for diagnosis and prognostic prediction.

\section{Effect of ELK3 on growth, invasion and migration of glioma cells}

Afterwards, we detected the expression of ELK3 in glioma cell lines and the effect of ELK3 expression on the growth and mobility of glioma cells. The data in Figure $3 \mathrm{~A}$ show that the levels of ELK3 in A172 and LN229 cells were 2.24- and 2.28-fold
B

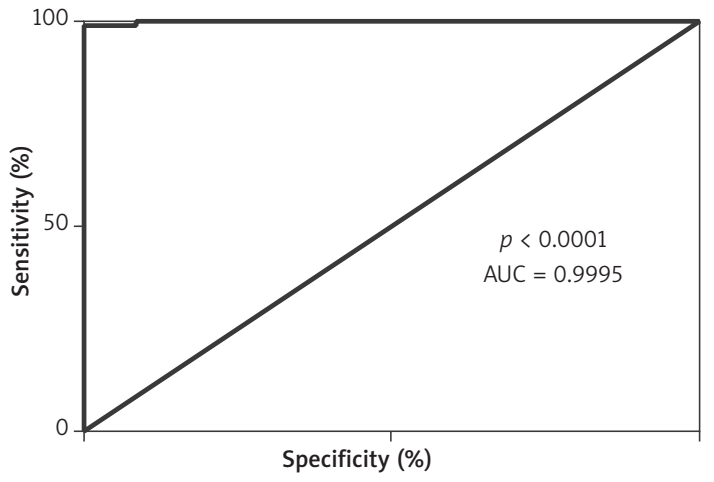

Figure 1. ELK3 was differentially expressed in tumors and had great value in glioma diagnosis. A - ELK3 expression was notably higher in glioma samples than the corresponding control, $p<0.0001$. B - Receiver operating characteristic (ROC) curve indicated that ELK3 had high diagnostic value, $p<0.0001$. C - Glioma patients in the high ELK3 expression group presented shorter overall survival than that in the low ELK3 expression group, $p=0.006$

those of NHA cells, respectively, indicating significantly higher expression of ELK3 in glioma cells than normal cells $(p<0.01)$. After transfection of siRNAs specific to ELK3, ELK3 expression was notably reduced in A172 and LN229 cells $(p<0.01)$. Si-ELK3\#2, with higher interference efficiency, was selected for further study (Figure $3 \mathrm{~B}-\mathrm{D}$ ). With siELK3 treatment, the OD values of A172 and LN229 cells were significantly lower than those in the sicon group at $48 \mathrm{~h}(p<0.05)$ and $72 \mathrm{~h}(p<0.01)$, indicating that depletion of ELK3 reduced the viability of A172 and LN229 cells (Figure 4 A-B). Data from Figure 4 C-E display that knockdown of ELK3 remarkably reduced the number of colonies formed by A172 cells (145.30 \pm 12.86 vs. 300.30

Table I. Information of datasets from CGGA database

\begin{tabular}{|lccccccc|}
\hline Dataset & Data type & \multicolumn{7}{c|}{ Sample number } \\
\cline { 3 - 8 } & & \#total & \#pLGG & \#rLGG & \#pGBM & \#rGBM & \#sGBM \\
\hline mRNAseq_325 & RNA-seq & 325 & 144 & 38 & 85 & 24 & 30 \\
\hline mRNAseq_693 & RNA-seq & 693 & 282 & 161 & 140 & 109 & 0 \\
\hline mRNA_array_301 & microarray & 301 & 156 & 18 & 108 & 5 & 11 \\
\hline
\end{tabular}


A

A $\quad \begin{aligned} \text { mRNAseq_325 } \\ p=4.8 \mathrm{e}-19\end{aligned}$

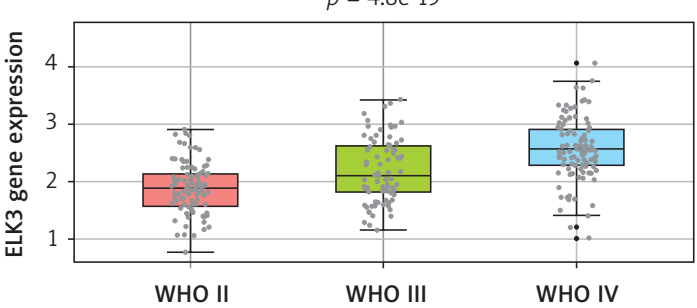

C
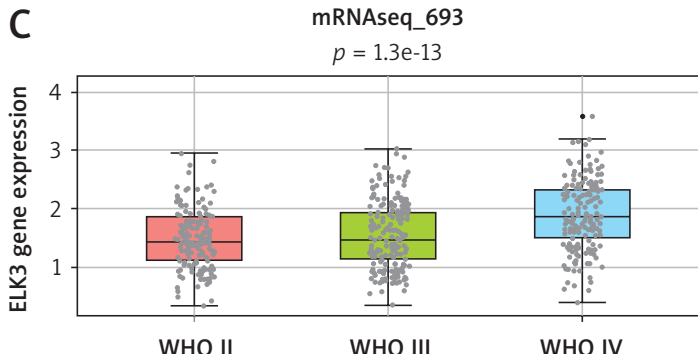

WHO II

mRNA_array_301

E

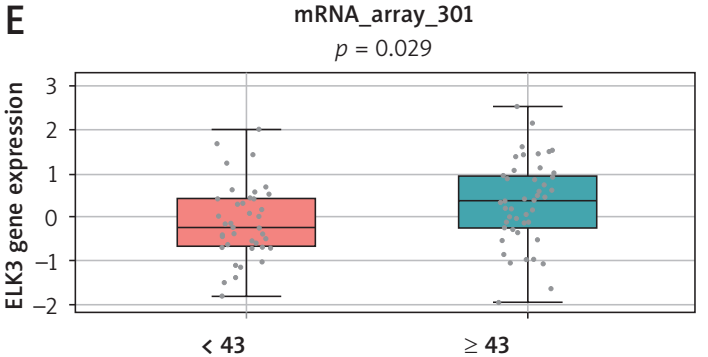

G

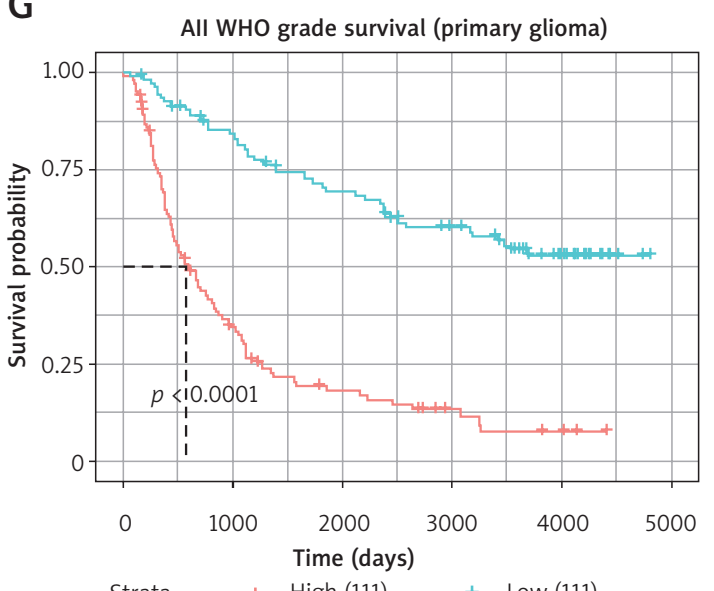

B MRNA array 301 $p=3.3 \mathrm{e}-06$

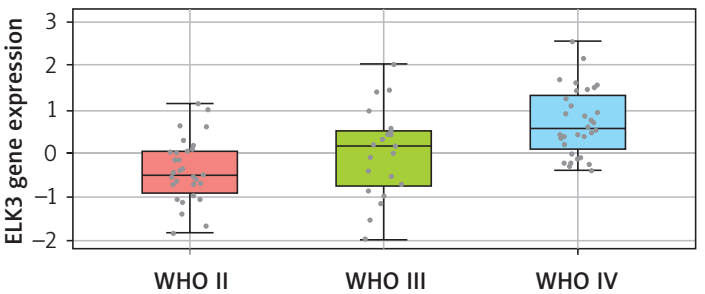

D mRNAseq_325
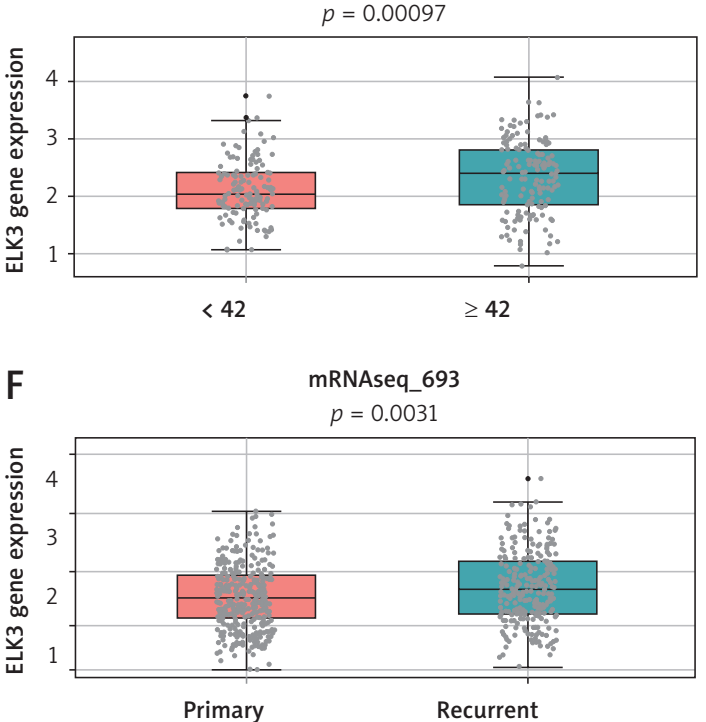

$\mathrm{H}$

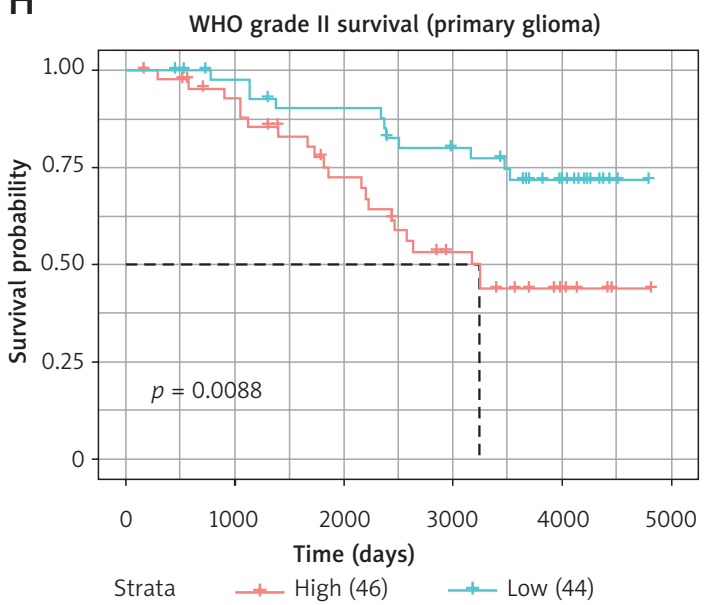

Figure 2. Expression of ELK3 in glioma patients with different WHO tumor grades, patients' ages and recurrent states. A-C - Data from mRNAseq_325, mRNAseq_693 and mRNA_array_301 datasets indicated that ELK3 expression was higher in WHO tumor grade IV than that in WHO tumor grades II and III. D, E - ELK3 expression was higher in older glioma patients. F - Compared with primary glioma, ELK3 expression was higher in recurrent glioma. G, H - In different WHO tumor grades of glioma and recurrent glioma, high expression of ELK3 led to a low survival rate. The numbers in parentheses represent the sample size

$\pm 11.68, p<0.01)$ and LN229 cells $(125.70 \pm 4.04$ vs. $256.00 \pm 20.07, p<0.01$ ) compared with the si-con group. Additionally, the numbers of invaded and migrated A172 cells (103.70 \pm 5.13 vs. 202.70 \pm 11.02 ; $148.30 \pm 3.26$ vs. $309.00 \pm 7.81 ; p<0.01)$ and LN229 cells $(104.30 \pm 3.21$ vs. $193.00 \pm 1.73$;
$142.00 \pm 3.61$ vs. $294.30 \pm 18.77 ; p<0.01$ ) were notably reduced when ELK3 was knocked down (Figure $4 \mathrm{~F}-\mathrm{I}$ ). All these findings suggest that depletion of ELK3 suppressed the malignant properties of glioma cells, further indicating the importance of ELK3 in glioma progression. 


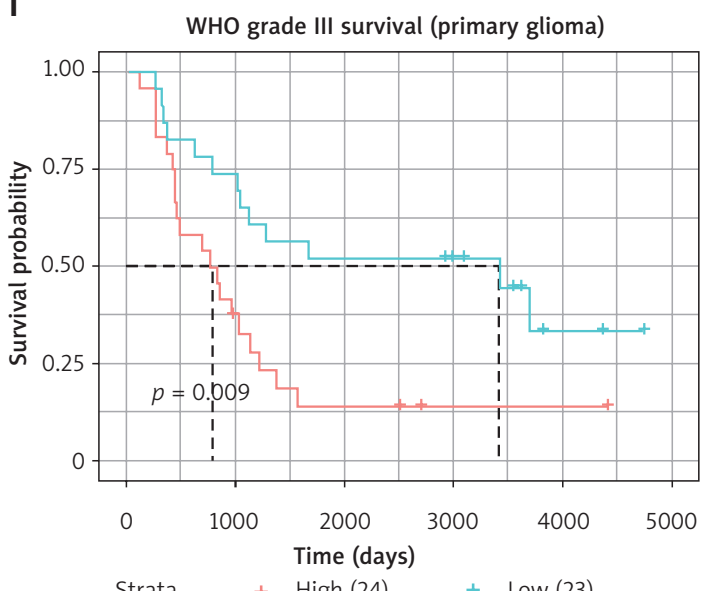

K

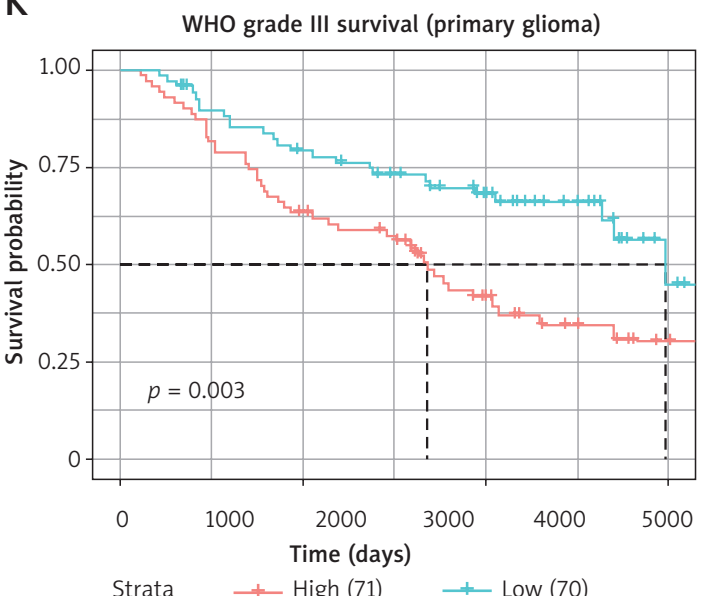

M

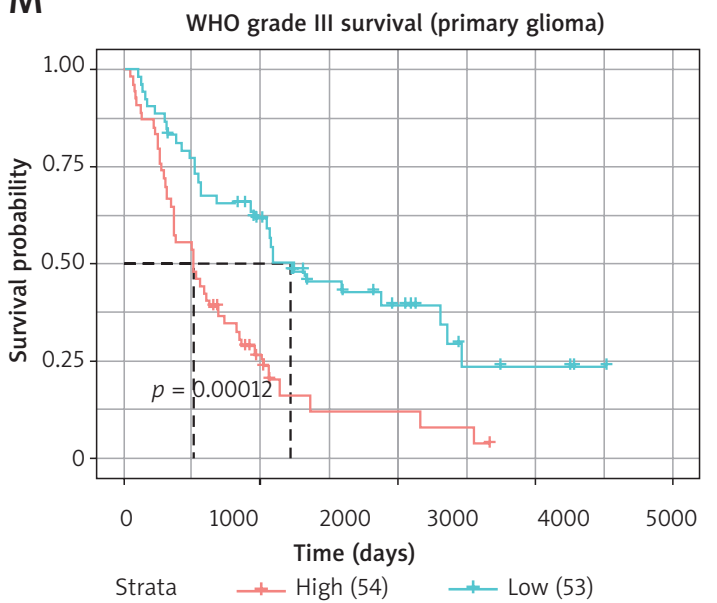

J

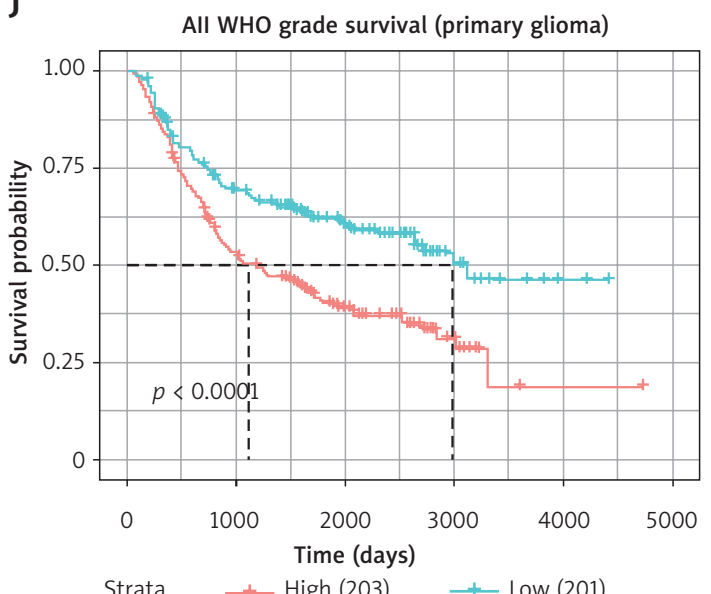

$\mathbf{L}$

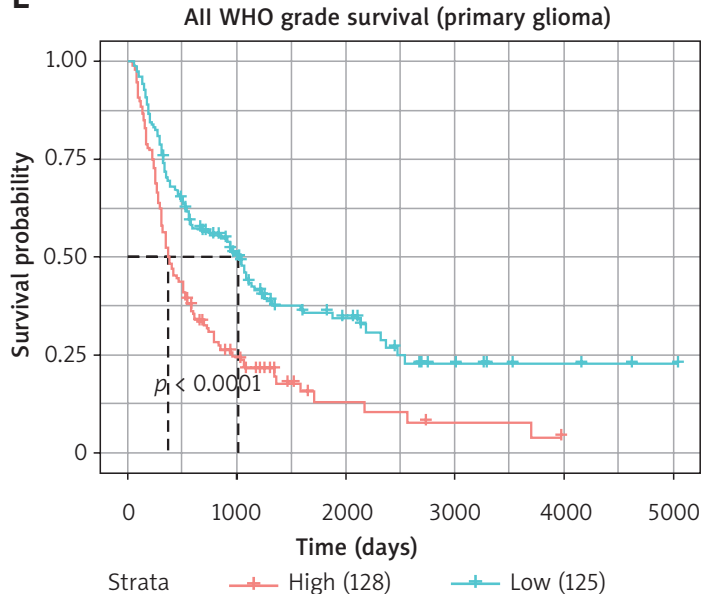

N

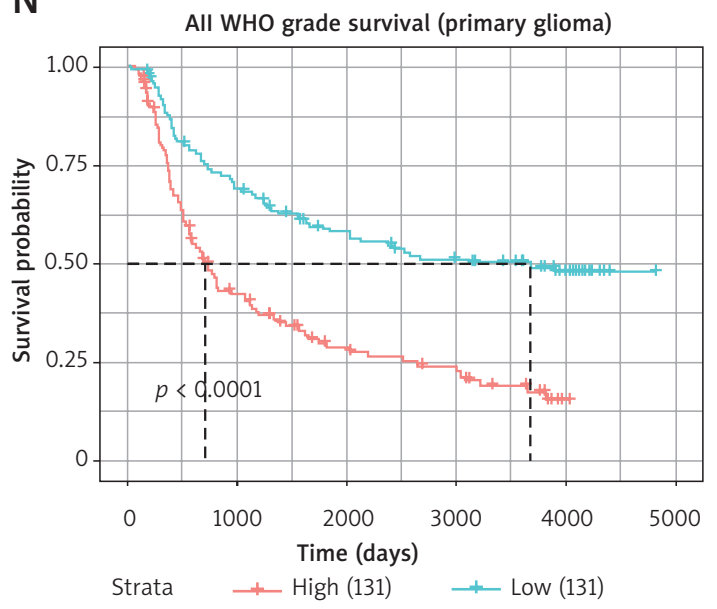

Figure 2. Cont. I-N - In different WHO tumor grades of glioma and recurrent glioma, high expression of ELK3 led to a low survival rate. The numbers in parentheses represent the sample size

\section{Effect of ELK3 on expression of EMT-associated proteins in glioma}

Through searching the GEPIA website, we discovered that ELK3 expression was positively associated with $\mathrm{N}$-cadherin $(R=0.21, p<0.01)$, Snail1 $(R=0.43, p<0.01)$, Slug $(R=0.6, p<0.01)$ and vimentin $(R=0.35, p<0.01)$ (Figure $5 \mathrm{~A}-\mathrm{D})$.
The results of Western blotting showed that knockdown of ELK3 notably suppressed the expression of $\mathrm{N}$-cadherin, Snail, Slug and vimentin in A172 and LN229 cells (Figure $5 \mathrm{E}-\mathrm{H}, p<0.01$ ), indicating that ELK3 possibly executed its function in glioma cells partly through regulating EMT progression. 
0

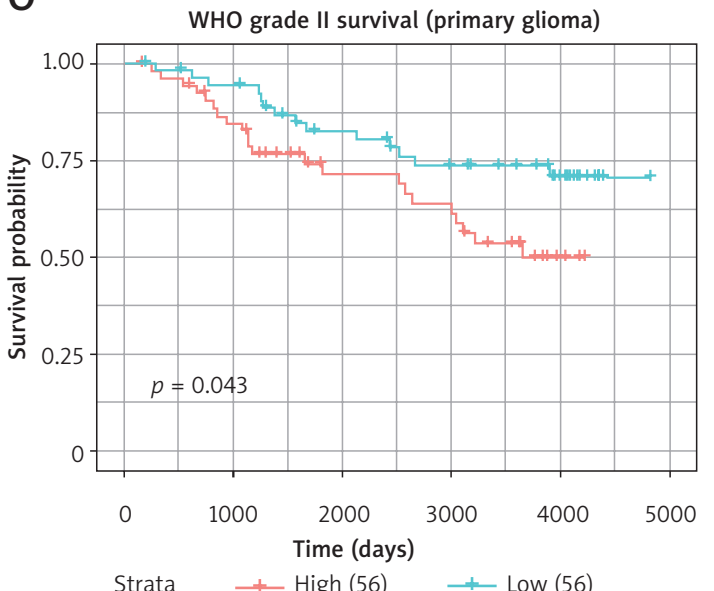

Q

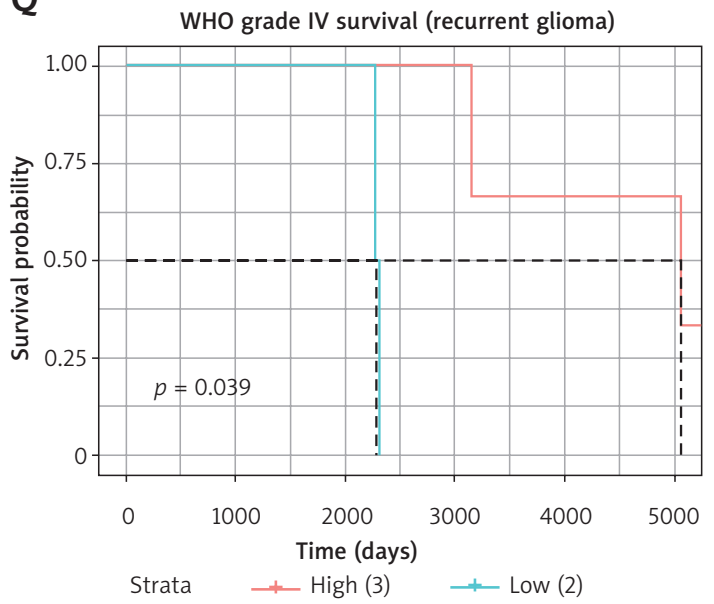

\section{Discussion}

Most glioma patients tend to present recurrence and poor prognosis. Although the treatment regimens have improved, the survival rates for glioma patients have barely changed [14]. Identifying reliable biomarkers with diagnostic and prognostic value and developing effective therapeutics are of great significance. In the present study, the results demonstrated for the first time that ELK3 was highly expressed in glioma samples and its high expression was connected with the poor prognosis of glioma patients. Besides that, knockdown of ELK3 suppressed the malignant properties of glioma cells and the progression of EMT. These findings suggested that ELK3 might be a novel diagnostic and prognostic marker as well as a therapeutic target for glioma.

ELK3, also named as Net, SAP-2, or Erp, is a protein containing the ETS domain that forms a ternary complex with DNA and serum response factor (SRF) [7], distinguished from other members of the ETS family by its ability to inhibit transcription [8]. ELK3 has been reported to contribute to the development and metastasis of breast cancer $[6,15]$ and facilitate the invasion and migration of liver

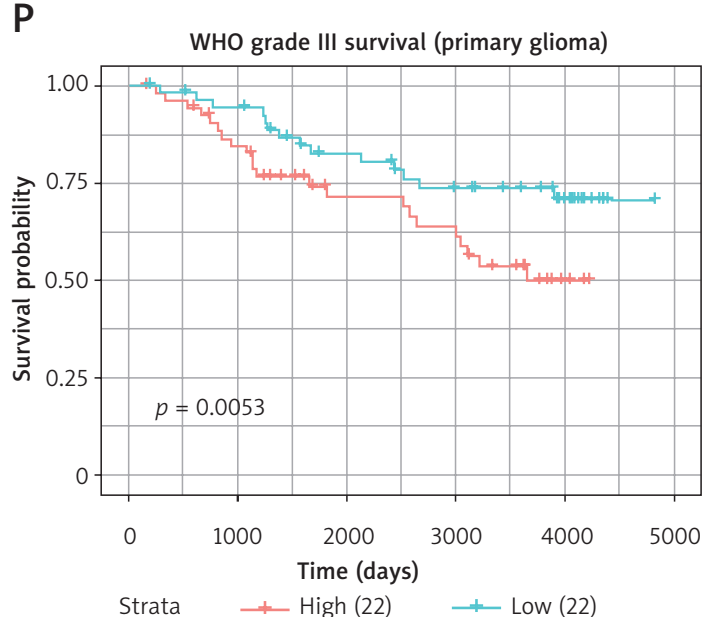

Figure 2. Cont. $\mathbf{O}-\mathbf{Q}-$ In different WHO tumor grades of glioma and recurrent glioma, high expression of ELK3 led to a low survival rate. The numbers in parentheses represent the sample size

cancer stem cells [7]. In addition, high ELK3 expression is revealed to be associated with gastric tumorigenesis and could enhance the infiltration of M2 macrophages [16]. All these data supported the critical role of ELK3 in the progression of several tumors. Herein, ELK3 was found to be up-regulated in glioma tissues and its high expression was correlated with poor prognosis. Age is a critical factor of cancer risk. The age of patients with gliomas is an independent prognostic factor for overall survival [18]. Of note, the results in this study showed that the expression of ELK3 was higher in older patients, suggesting the relation of ELK3 expression and the age of patients with gliomas. Moreover, the biological experiments in the present study illustrated that depletion of ELK3 suppressed the proliferation and mobility of glioma cells. These findings consistently suggested the promoting role of ELK3 during glioma progression.

A better understanding of the molecular mechanism of glioma growth and motility is necessary to enhance the efficacy of clinical therapy in inhibiting glioma metastasis. EMT induces cells to lose their epithelial properties, achieve invasive and migratory capacity and change from an epithelial cell community to mesenchymal cells [17, 18]. 
A

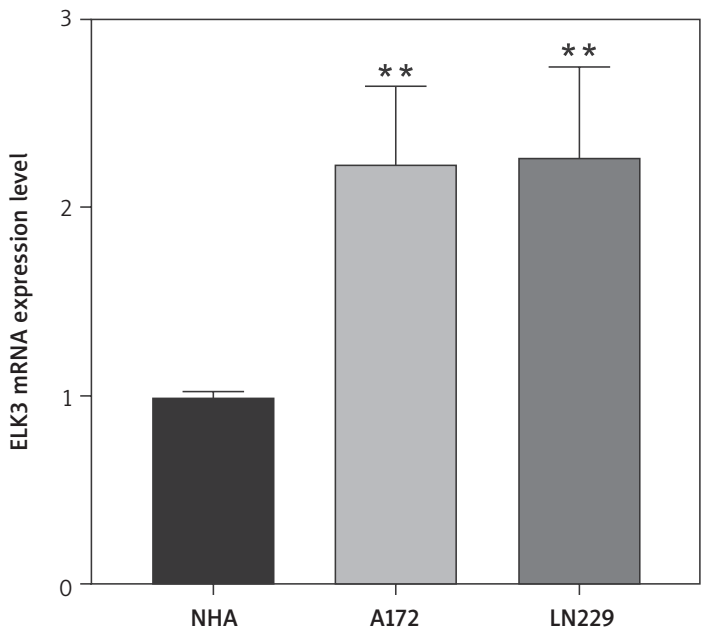

C

si-con si-ELK3\#1 si-ELK3\#2 si-con si-ELK3\#1 si-ELK3\#2

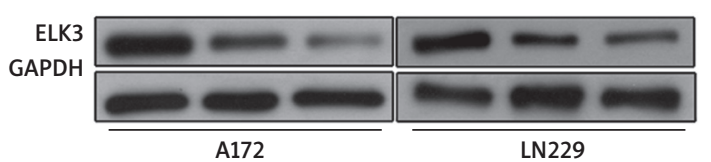

B

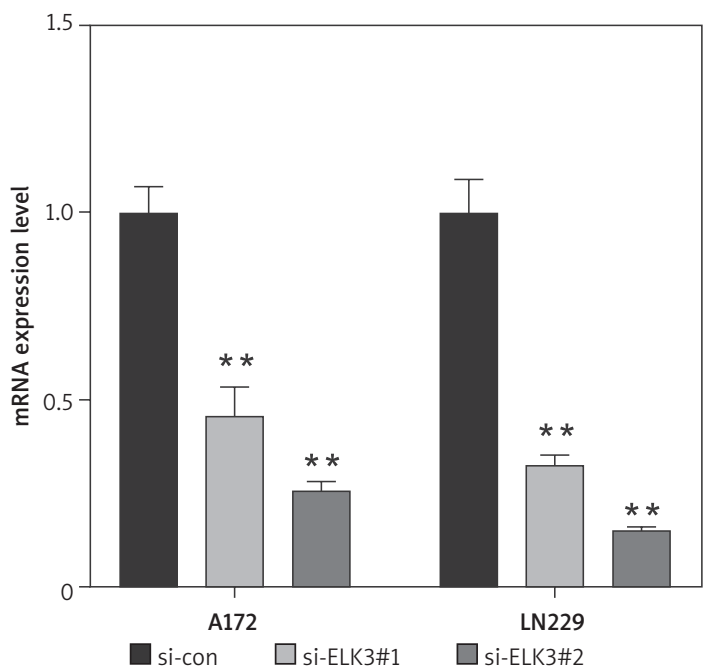

D

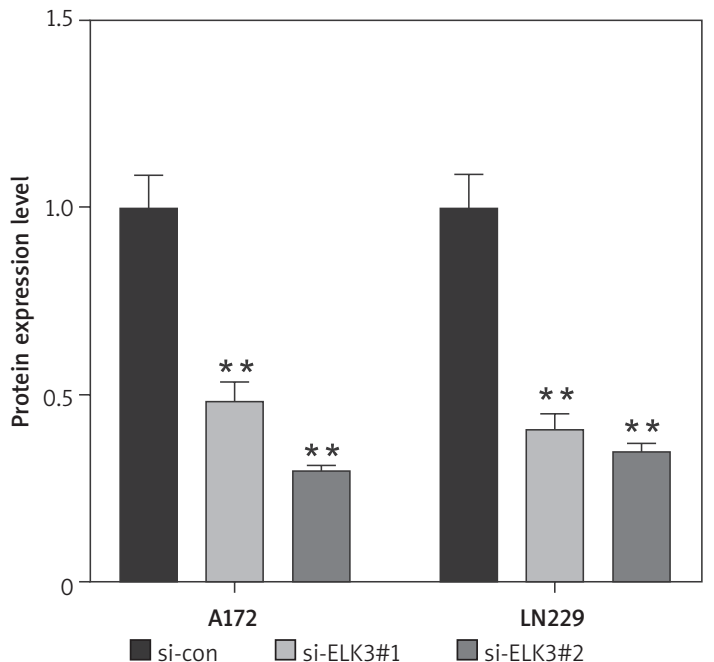

Figure 3. ELK3 expression was increased in glioma cells and reduced with si-ELK3 treatment. A - Expression of ELK3 was higher in A172 and LN229 cells compared with NHA cells, ${ }^{* *} p<0.01$. B-D - The mRNA and protein expression levels of ELK3 were notably reduced in A172 and LN229 cells when treated by si-ELK3\#1 or si-ELK3\#2, ${ }^{* *} p<0.01$ vs. si-con

As a key factor of tumor invasion enhancement, EMT has been illustrated to be implicated in the malignant development of glioma $[19,20]$. Moreover, we know that tumor cells and stromal components cooperatively participate in the malignant development and recurrence of glioma [19]. Just because of this, the study of EMT in glioma is crucial to reverse malignant development and reduce recurrence. It has been reported that ELK3 plays a central role in the EMT process, leading to liver fibrosis through the MAPK pathway [21]. Previously, a study revealed that inhibition of ELK3 impaired the ability of TGF $\beta$ signaling to activate the expression of mesenchymal markers such as Snail, vimentin, and Slug in breast cancer cells $[22,23]$. Analysis from the GEPIA website displayed that ELK3 expression was positively related with N-cadherin, Snail, Slug and vimentin. Moreover, the biological experiments further identified the associations between ELK3 and EMT-related markers, which showed that knockdown of ELK3 suppressed the expression of N-cadherin, Snail, Slug and vimentin in glioma cells. These observations indicated that ELK3 promoted the progression of glioma at least partially by activating the EMT process.

In conclusion, ELK3, which is highly expressed in glioma, is predictive of a poor prognosis for patients with glioma. Furthermore, ELK3 contributed to the growth and mobility of glioma cells partly through regulation of the EMT process, indicating that inhibition of ELK3 expression might be a valuable therapeutic strategy for preventing progression of tumor growth and metastasis. 
A

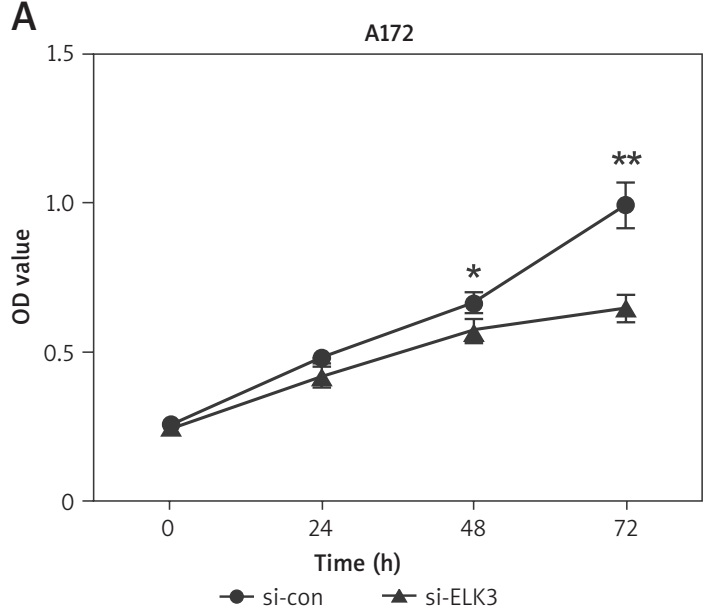

C

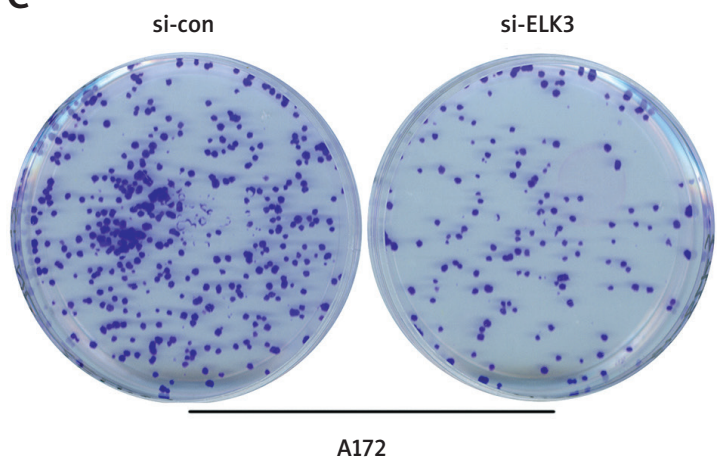

E

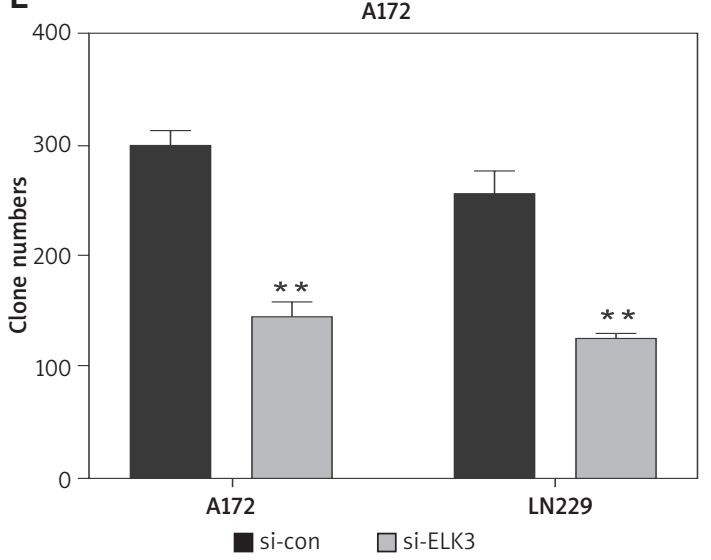

G

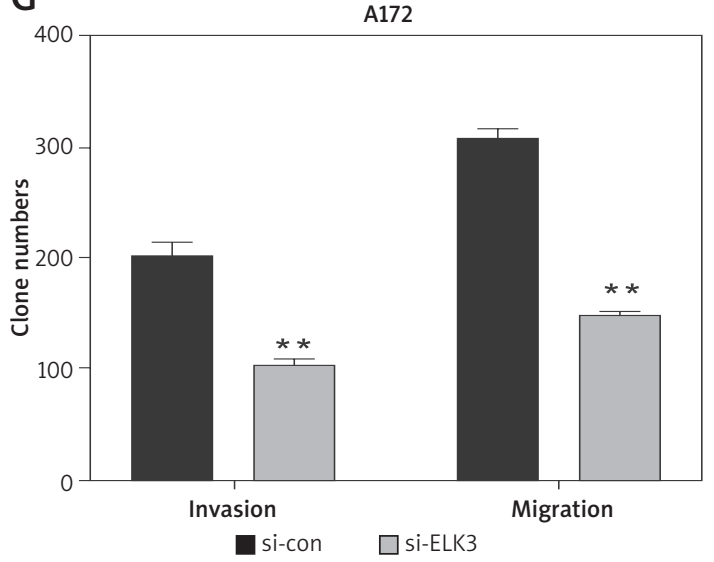

B

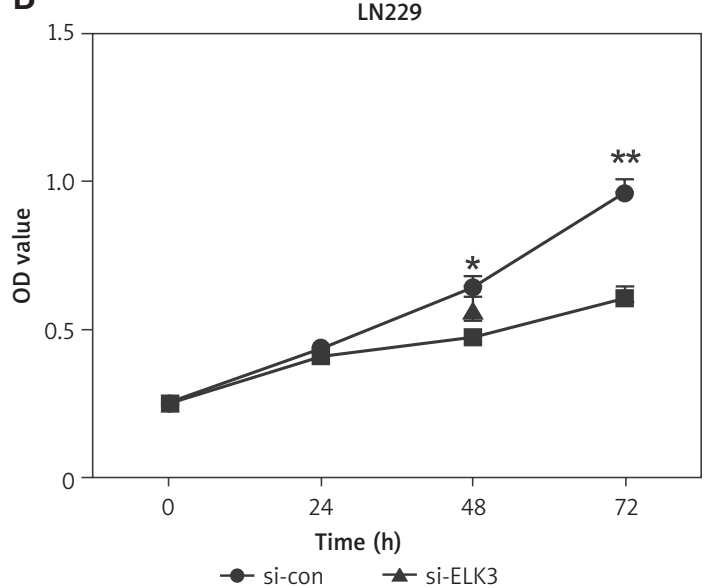

D

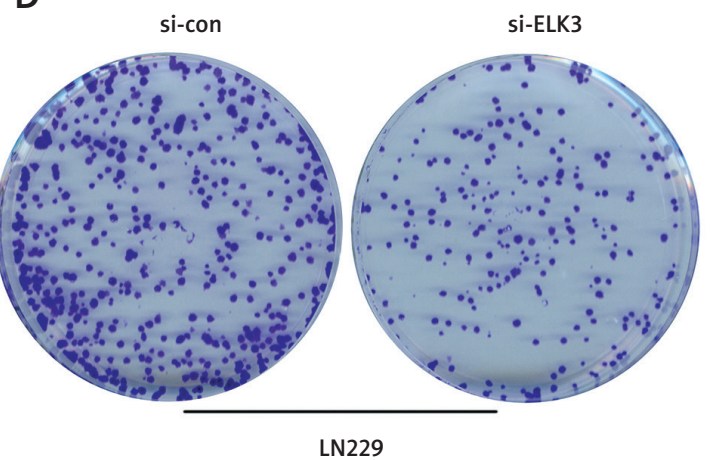

F si-con si-ELK3

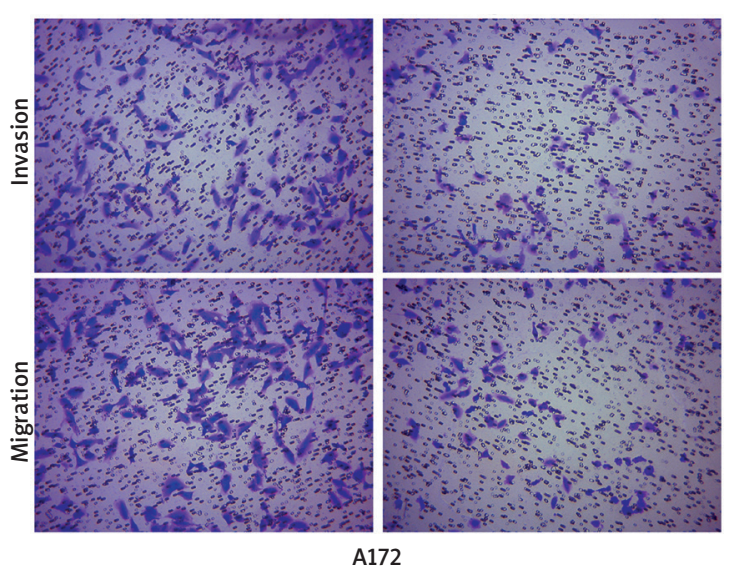

Figure 4. Malignant behaviors of glioma cells were suppressed by si-ELK3 treatment. A, B - The OD values of A172 or LN229 cells were notably reduced when ELK3 was knocked down. C-E - Numbers of colonies formed by A172 and LN229 cells were reduced significantly by si-ELK3 treatment. F, G - The numbers of invaded and migrated A172 or LN229 cells were reduced obviously after si-ELK3 treatment. ${ }^{*} p<0.05,{ }^{* *} p<0.01$ vs. si-con 

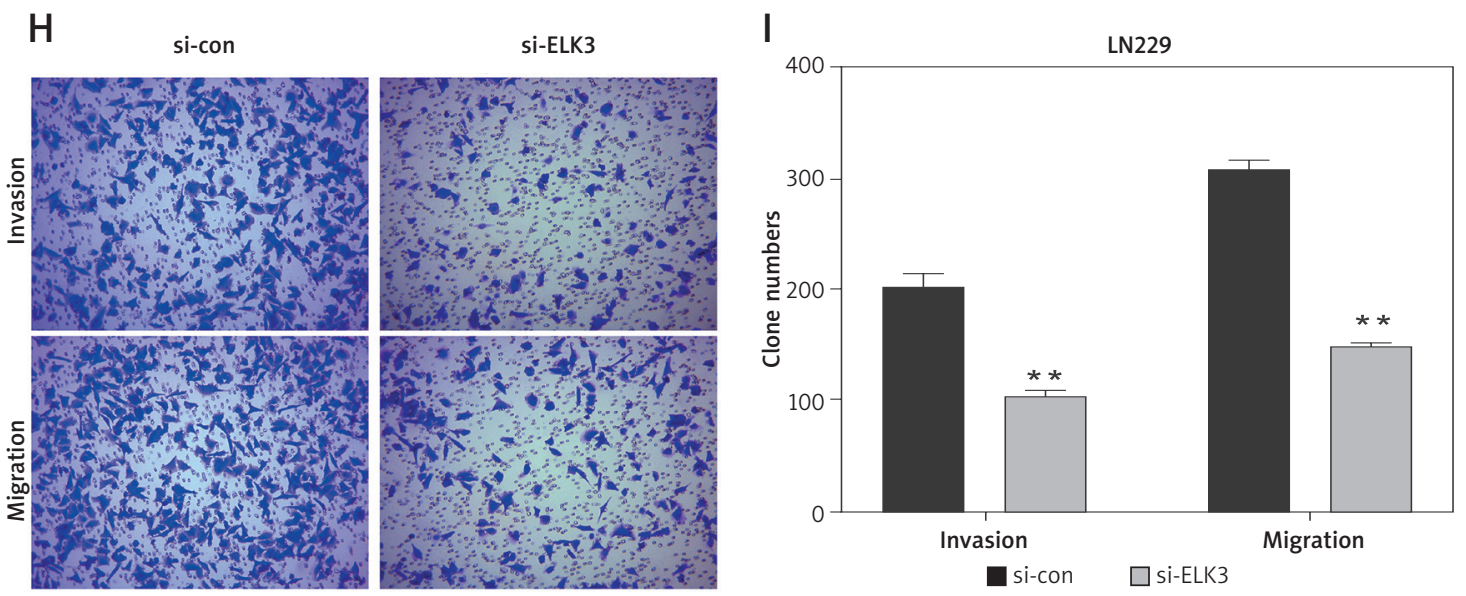

Figure 4. Cont. H, I-The numbers of invaded and migrated A172 or LN229 cells were reduced obviously after si-ELK3 treatment. ${ }^{*} p<0.05,{ }^{* *} p<0.01$ vs. si-con

A

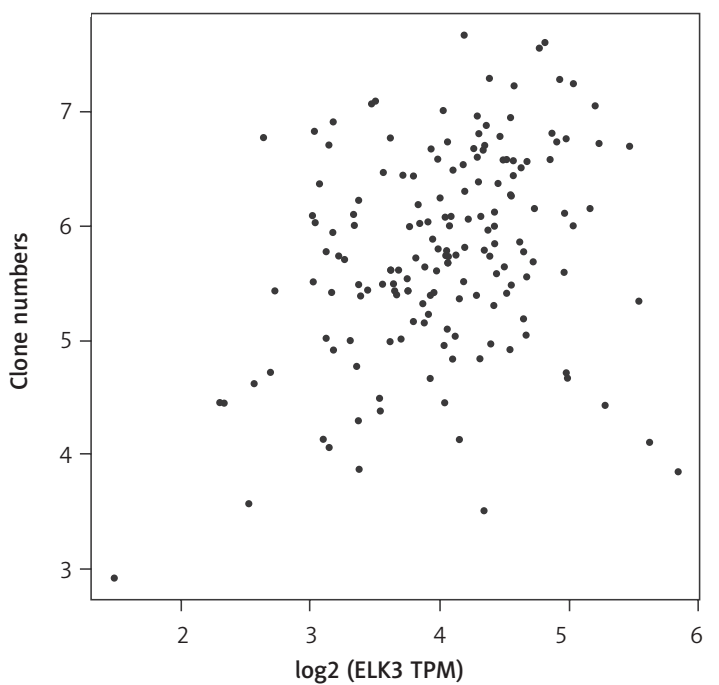

C

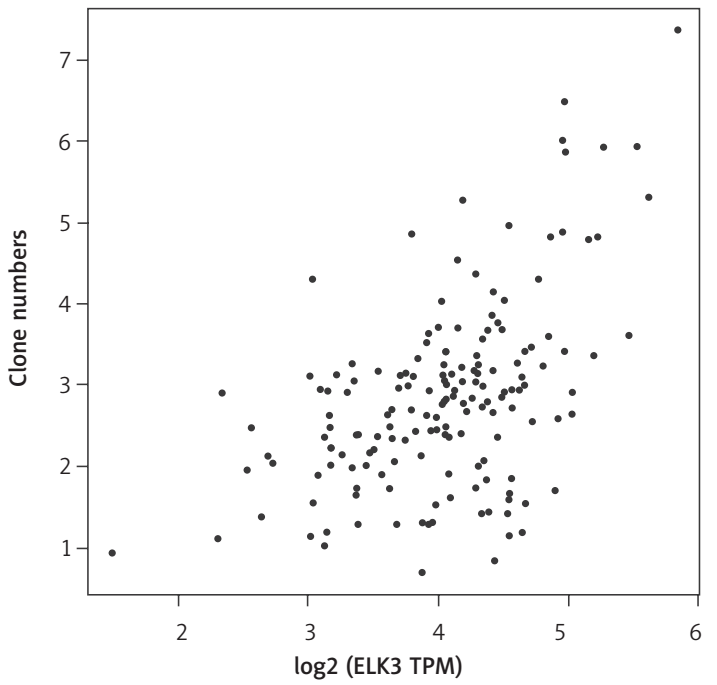

B

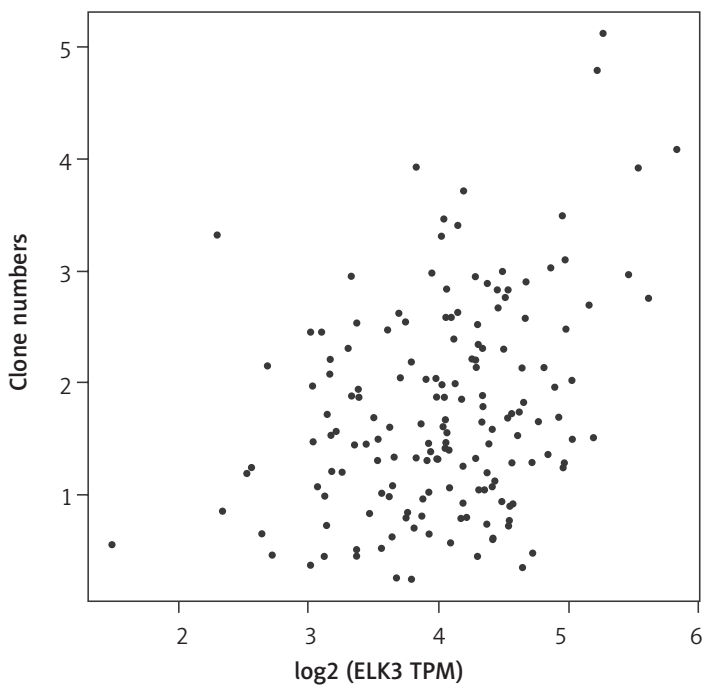

D

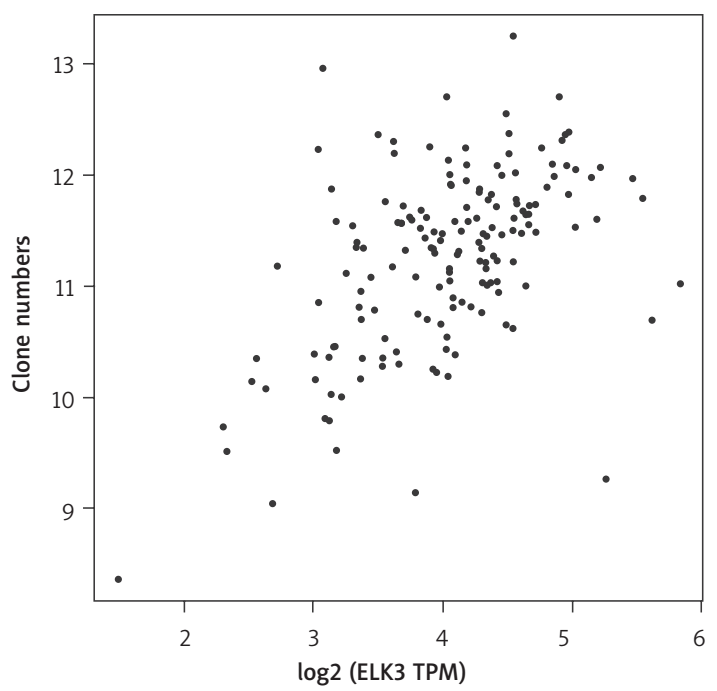

Figure 5. EMT-related genes were regulated by ELK3 in glioma. Analysis from GEPIA indicated that ELK3 expression was positively related to $\mathrm{N}$-cadherin (A), Snail1 (B), Slug (C) and vimentin (D). ${ }^{* *} p<0.01$ vs. si-con 
E

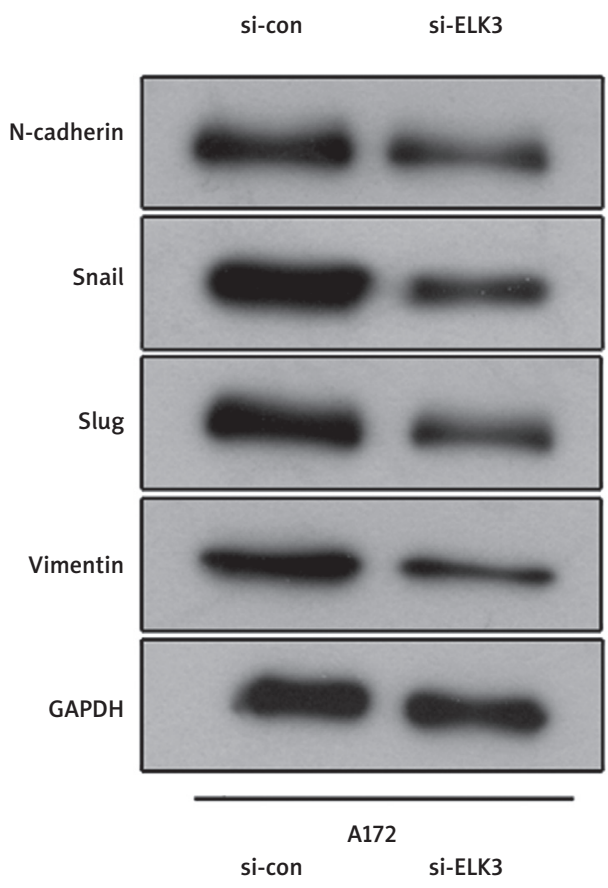

G

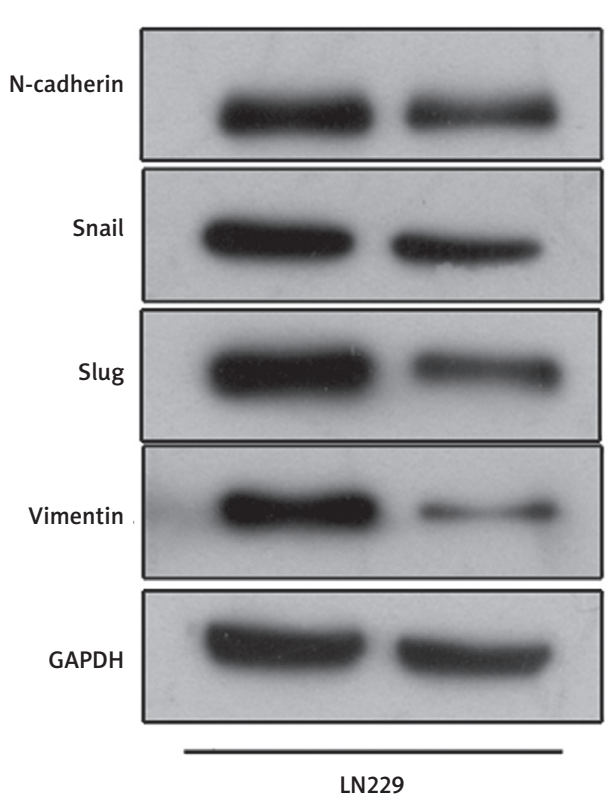

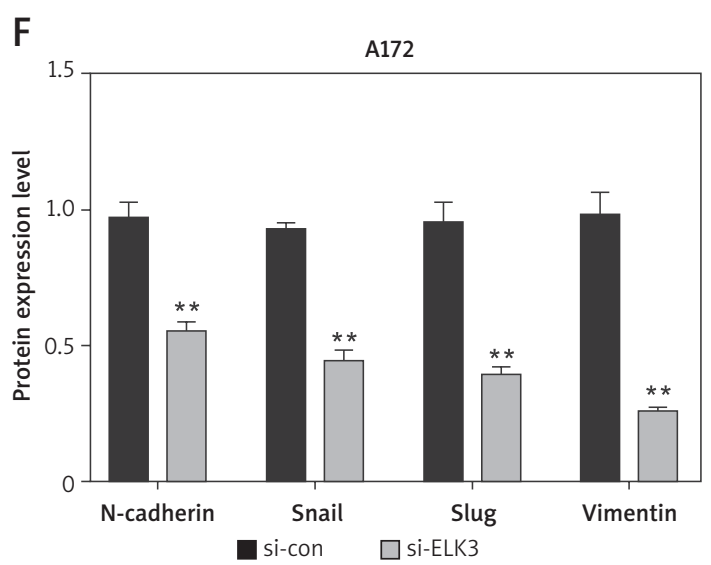

$\mathrm{H}$

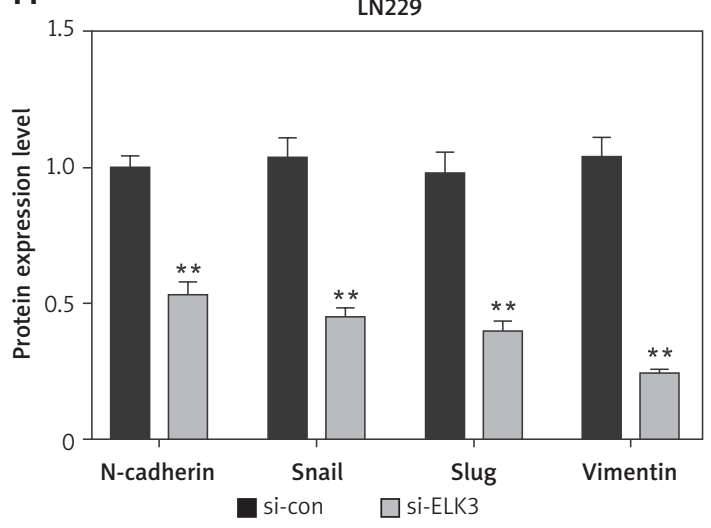

Figure 5. Cont. E, F - In A172 cells, knockdown of ELK3 reduced the protein expression of N-cadherin, Snail, Slug and vimentin. G, H - Knockdown of ELK3 suppressed the expression of N-cadherin, Snail, Slug and vimentin in LN229 cells. ${ }^{* *} p<0.01$ vs. si-con

\section{Acknowledgments}

Jiang-tao Dong and Hui Xv contributed equally to this work.

\section{Conflict of interest}

The authors declare no conflict of interest.

\section{References}

1. Chen R, Smith-Cohn M, Cohen AL, Colman H. Glioma subclassifications and their clinical significance. Neurotherapeutics 2017; 14: 284-97.
2. Modrek AS, Bayin NS, Placantonakis DG. Brain stem cells as the cell of origin in glioma. World I Stem Cells 2014; 6: 43-52.

3. Wang J, Su HK, Zhao HF, Chen ZP, To SS. Progress in the application of molecular biomarkers in gliomas. Biochem Biophys Res Commun 2015; 465: 1-4.

4. Wang H, LiU G, Li T, Wang N, Wu J, Zhi H. MiR-330-3p functions as a tumor suppressor that regulates glioma cell proliferation and migration by targeting CELF1. Arch Med Sci 2020; 16: 1166-75.

5. Liu DK, Wei YJ, Guo Y, Wang J, Wang GH. MiRNA-93 functions as an oncogene in glioma by directly targeting RBL2. Eur Rev Med Pharmacol Sci 2018; 22: 2343-50. 
6. Kim KS, Park J, Oh N, Cho HJ, Park JH, Park KS. ELK3 expressed in lymphatic endothelial cells promotes breast cancer progression and metastasis through exosomal miRNAs. Sci Rep 2019; 9: 8418.

7. Lee JH, Hur W, Hong SW, et al. ELK3 promotes the migration and invasion of liver cancer stem cells by targeting HIF-1 $\alpha$. Oncol Rep 2017; 37: 813-22.

8. Zheng H, Wasylyk C, Ayadi A, et al. The transcription factor Net regulates the angiogenic switch. Genes Dev 2003; 17: 2283-97.

9. Li TZ, Kim SM, Hur W, et al. Elk-3 contributes to the progression of liver fibrosis by regulating the epithelialmesenchymal transition. Gut Liver 2017; 11: 102-11.

10. Lu J, Li D, Zeng Y, et al. IDH1 mutation promotes proliferation and migration of glioma cells via EMT induction. J BUON 2019; 24: 2458-64.

11. Noh MG, Oh SJ, Ahn EJ, et al. Prognostic significance of $\mathrm{E}$-cadherin and $\mathrm{N}$-cadherin expression in gliomas. BMC Cancer 2017; 17: 583.

12. Gonzalez DM, Medici D. Signaling mechanisms of the epithelial-mesenchymal transition. Sci Signal 2014; 7: re8.

13. Cho HJ, Oh N, Park JH, et al. ZEB1 collaborates with ELK3 to repress $\mathrm{E}$-Cadherin expression in triple-negative breast cancer cells. Mol Cancer Res 2019; 17: 2257-66.

14. Zhang J, Cai H, Sun L, et al. LGR5, a novel functional glioma stem cell marker, promotes EMT by activating the Wnt/ $\beta$-catenin pathway and predicts poor survival of glioma patients. J Exp Clin Cancer Res 2018; 37: 225

15. Heo SH, Lee JY, Yang KM, Park KS. ELK3 expression correlates with cell migration, invasion, and membrane type 1-matrix metalloproteinase expression in MDA-MB-231 breast cancer cells. Gene Expr 2015; 16: 197-203.

16. Dazhi W, Zheng J, Chunling R. High ELK3 expression is associated with the VEGF-C/VEGFR-3 axis and gastric tumorigenesis and enhances infiltration of $\mathrm{M} 2$ macrophages. Future Med Chem 2020; 12: 2209-24.

17. Kalluri R, Weinberg RA. The basics of epithelial-mesenchymal transition. J Clin Invest 2009; 119: 1420-8.

18. Xu J, Lamouille S, Derynck R. TGF-beta-induced epithelial to mesenchymal transition. Cell Res 2009; 19: 156-72.

19. Karsy M, Gelbman M, Shah P, Balumbu O, Moy F, Arslan E. Established and emerging variants of glioblastoma multiforme: review of morphological and molecular features. Folia Neuropathol 2012; 50: 301-21.

20. Dong N, Guo J, Han S, Bao L, Diao Y, Lin Z. Positive feedback loop of IncRNA HOXC-AS2/miR-876-5p/ZEB1 to regulate EMT in glioma. Onco Targets Ther 2019; 12: 7601-9.

21. Li TZ, Kim SM, Hur W, et al. Elk-3 contributes to the progression of liver fibrosis by regulating the epithelialmesenchymal transition. Gut Liver 2017; 11: 102-11.

22. Kong SY, Kim KS, Kim J, et al. The ELK3-GATA3 axis orchestrates invasion and metastasis of breast cancer cells in vitro and in vivo. Oncotarget 2016; 7: 65137-46.

23. Kim KS, Kim J, Oh N, Kim MY, Park KS. ELK3-GATA3 axis modulates MDA-MB-231 metastasis by regulating cellcell adhesion-related genes. Biochem Biophys Res Commun 2018; 498: 509-15. 